\title{
The Involvement of PDE4 in the Protective Effects of Melatonin on Cigarette-Smoke-Induced Chronic Obstructive Pulmonary Disease
}

\author{
Je-Oh Lim ${ }^{1,+}$, Woong-Il Kim ${ }^{1,+}$, Se-Jin Lee ${ }^{1}$, So-Won Pak ${ }^{1}$, Young-Kwon Cho ${ }^{2}$, Jong-Choon Kim ${ }^{1}$, \\ Joong-Sun Kim ${ }^{1}$ and In-Sik Shin ${ }^{1, *}$ \\ 1 BK21 FOUR Program, College of Veterinary Medicine, Chonnam National University, 77 Yongbong-ro, \\ Buk-gu, Gwangju 61186, Korea; 166634@jnu.ac.kr (J.-O.L.); 208521@jnu.ac.kr (W.-I.K.); \\ 218729@jnu.ac.kr (S.-J.L.); 208514@jnu.ac.kr (S.-W.P.); toxkim@jnu.ac.kr (J.-C.K.); centraline@jnu.ac.kr (J.-S.K.) \\ 2 College of Health Science, Cheongju University, Chungbuk 28503, Korea; petmen@hanmail.net \\ * Correspondence: dvmshin@jnu.ac.kr; Tel.: +82-62-530-2835 \\ + These authors contributed equally to this work.
}

check for

updates

Citation: Lim, J.-O.; Kim, W.-I.; Lee, S.-J.; Pak, S.-W.; Cho, Y.-K.; Kim, J.-C.; Kim, J.-S.; Shin, I.-S. The Involvement of PDE4 in the Protective Effects of Melatonin on Cigarette-SmokeInduced Chronic Obstructive Pulmonary Disease. Molecules 2021, 26, 6588. https://doi.org/10.3390/ molecules 26216588

Academic Editor: Andrzej Slominski

Received: 6 October 2021

Accepted: 28 October 2021

Published: 30 October 202

Publisher's Note: MDPI stays neutral with regard to jurisdictional claims in published maps and institutional affiliations.

Copyright: (c) 2021 by the authors. Licensee MDPI, Basel, Switzerland. This article is an open access article distributed under the terms and conditions of the Creative Commons Attribution (CC BY) license (https:// creativecommons.org/licenses/by/ $4.0 /)$.

\begin{abstract}
Chronic obstructive pulmonary disease (COPD) is a significant disease threatening human health. Currently, roflumilast, a phosphodiesterase (PDE) 4 inhibitor, is recommended as a therapeutic agent for COPD. In this study, we investigated the therapeutic effects of melatonin against COPD, focusing on determining whether it is a PDE4 inhibitor via in vivo and in vitro experiment using cigarette smoke (CS) and cigarette smoke condensate (CSC), respectively. In the in vivo experiments, melatonin treatment reduced inflammatory responses, including inflammatory cell counts. Melatonin treatment also suppressed the CS-exposure-induced upregulation of cytokine and matrix metalloproteinase (MMP)-9, reduced the PDE4B expression, and elevated cAMP levels. In addition, these effects were synergistic, as melatonin and roflumilast cotreatment eventually ameliorated the CS-exposure-induced worsening of lung function. In the CSC-stimulated NCI-H292 cells, melatonin inhibited elevation in the levels of inflammatory cytokines, MMP-9, and PDE4, and elevated cAMP levels. Furthermore, melatonin and roflumilast cotreatment was more effective on inflammatory responses than only melatonin or roflumilast treatment. Our results indicate that melatonin relieves inflammatory response and loss of lung function in COPD, which is associated with decreased PDE4 expression. Therefore, we suggest that melatonin is a putative candidate for the treatment of COPD.
\end{abstract}

Keywords: melatonin; chronic obstructive pulmonary disease; cigarette smoke; phosphodiesterase 4; MMP-9

\section{Introduction}

The prevalence and mortality associated with chronic obstructive pulmonary disease (COPD) continues to increase worldwide. It is thus regarded as an important disease threatening human health [1]. COPD is characterized by pulmonary inflammation, bronchoconstriction, and mucus production, which eventually induce airflow restrictions due to loss of elastic recoil of the pulmonary tract [2]. Cigarette smoke (CS) is regarded as a crucial cause for the development of COPD, and it generates various stimuli, such as proinflammatory mediators and oxidative stress, resulting in pulmonary inflammation [3]. Continuous smoking increases the incidence of COPD via the elevation of inflammatory responses and oxidative damage. Therefore, inhibition of the inflammation and oxidative damage induced by CS is considered an important strategy for the effective control of COPD [4].

Loss of lung function caused by CS is closely associated with cyclic adenosine monophosphate (cAMP) and cyclic guanosine monophosphate (cGMP) [5]. Continuous smoking increases the expression of phosphodiesterase (PDE) in inflammatory cells, including neutrophils and macrophages, which promotes the conversion of cAMP to AMP, resulting 
in the decline of normal lung function [6-8]. Therefore, the inhibition of PDE expression ameliorates the CS-induced worsening of lung function via the elevation of cAMP levels. Based on these observations, the use of PDE inhibitors as therapeutic drugs has been recommended for COPD treatment [9]. Among PDE inhibitors, roflumilast is the first PDE4 inhibitor to be approved as a therapeutic drug to reduce the deterioration due to COPD [10]. However, as roflumilast has systemic side effects such as headache, weight loss, and vomiting, it is necessary to develop a therapeutic agent that can overcome these side effects [11].

Melatonin is a hormone secreted mainly from the pineal gland and has various pharmacological properties including anti-inflammatory, antioxidant, anti-apoptotic, and anti-tumor effects $[12,13]$. Melatonin is also generated in the peripheral organs, as well as the pineal gland, and produces its metabolites through a specific metabolism, which contributes to the maintenance of the proper function of the peripheral organs [14-16]. Due to the pharmacological properties of melatonin, protective and ameliorative effects by melatonin are observed in various respiratory diseases. In particular, we have demonstrated that melatonin alleviates lung inflammation induced by CS $[17,18]$. Melatonin reduced cigarette smoke condensate (CSC)-induced upregulation of mucin 5AC (MUC5AC) expression in human lung epithelial cells through suppression of mitogen-activated protein kinase signaling [17] and decreased the neutrophilic inflammatory response and mucin secretion induced by CS and lipopolysaccharide (LPS) through Erk-Sp1 signaling [18]. In addition, melatonin attenuated the pathophysiological condition of COPD via enhancement of SIRT1 expression and decreased the production of interleukin (IL)-8 induced by CS in pulmonary fibrosis [19-21]. Moreover, in clinical trials, melatonin reduced lung oxidative stress in patients with COPD [22]. However, the potential of melatonin as a PDE4 inhibitor in the treatment of COPD has not been examined.

We investigated the potential of melatonin as a PDE4 inhibitor in the treatment of COPD by exposing mice to CS and stimulating airway epithelial cells by cigarette smoke condensate (CSC). In addition, we studied the synergistic effects of melatonin and roflumilast, which is recommended as a PDE4 inhibitor in the treatment of COPD.

\section{Results}

\subsection{Effect of Melatonin on Inflammatory Mediators in Mice Exposed to CS}

Inflammatory cell count in the BALF of the CS group was higher than that in the BALF of the NC (Figure 1). However, the mice from the ROF and MEL groups exhibited significant reduction in inflammatory cell counts in BALF compared to those in the CS group. In particular, the counts were markedly reduced in the RM group (roflumilast and melatonin cotreatment) compared to those in the ROF or MEL groups.

Histological examination of the lung tissue revealed extensive inflammatory infiltration into the lung tissue of mice from the CS group (Figure 2). By contrast, ROF and MEL treatments decreased the pulmonary inflammation induced by exposure to CS. In addition, reduction in pulmonary inflammation was reduced to a greater extent in the RM group than in the ROF or MEL groups.

IL-6 levels in BALF were higher in samples from the CS group than the NC group (Figure 3a). In contrast, ROF and MEL groups showed significantly decreased levels of IL-6 in BALF, compared to the CS group. The reduction in IL-6 levels was the most noticeable in the RM group. Similar to the IL-6 levels, the TNF- $\alpha$ levels in BALF were higher in the CS group than in the NC group (Figure 3b). However, the ROF, MEL, and RM groups showed significantly lower TNF- $\alpha$ levels in BALF than the CS group, with the most remarkable decrease in the RM group. 
口NC घCS GROF घMEL DRM

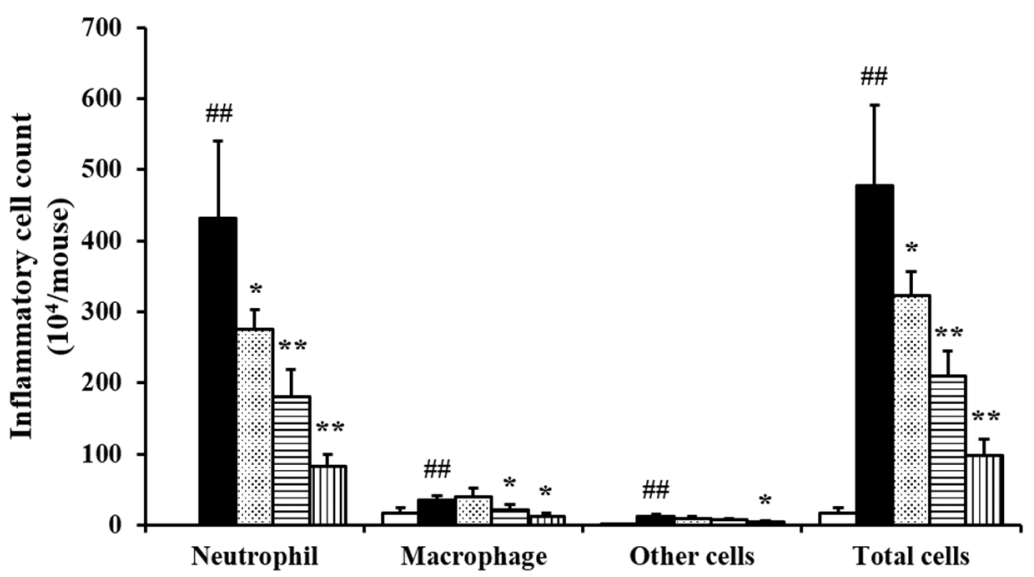

Figure 1. Melatonin decreased inflammatory cell counts in BALF of CS-exposed mice. Cells was determined by Diff-Quik ${ }^{\circledR}$ staining and counted using a light microscope. NC: non-treated and non-exposure group; CS: PBS-treated and CS+LPS exposure group; ROF: roflumilast-treated and CS+LPS exposure group, MEL: melatonin-treated and CS+LPS exposure group, RM: roflumilast and melatonin co-treated and CS+LPS exposure group. The data are expressed as mean \pm SD $(n=5)$. \#\# Significantly different from the NC group, $p<0.01 ;{ }^{*}, * *$ significantly different from the CS group, $p<0.05$ or $<0.01$, respectively.

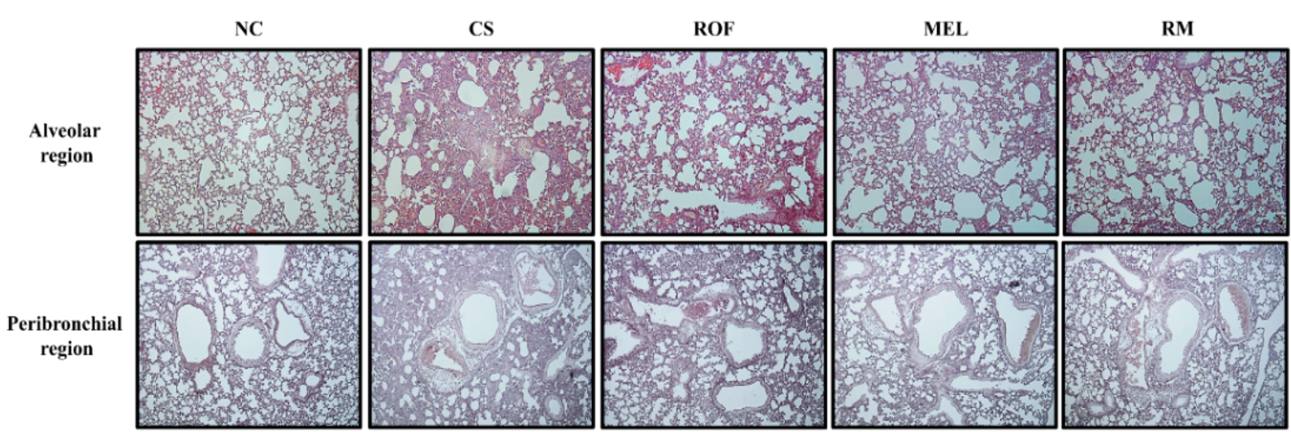

(a)

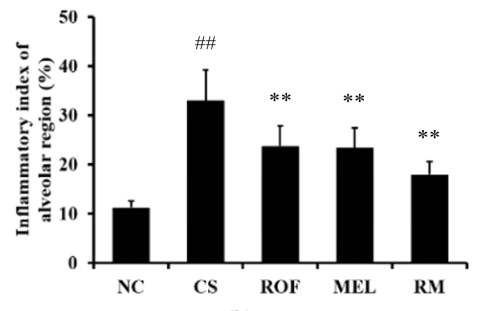

(b)

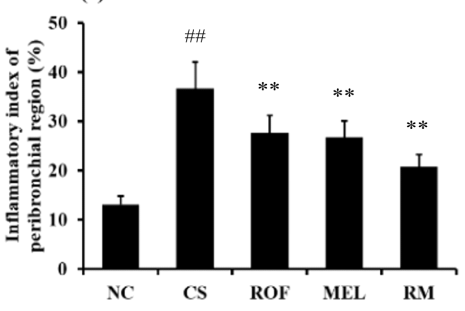

(c)

Figure 2. Melatonin reduced inflammatory cell infiltration in lung tissue of CS-exposed mice. The inflammatory cells infiltration was determined by H\&E staining $(n=5)$. (a) lung histology stained with H\&E, (b) inflammatory index of alveolar region, (c) inflammatory index of peribronchial region. NC: non-treated and non-exposure group; CS: PBS-treated and CS+LPS exposure group; ROF: roflumilast-treated and CS+LPS exposure group, MEL: melatonin-treated and CS+LPS exposure group, RM: roflumilast and melatonin co-treated and CS+LPS exposure group. The data are expressed as mean $\pm S D(n=5)$. ${ }^{\#}$ Significantly different from the NC group, $p<0.01 ;{ }^{* *}$ significantly different from the CS group, $p<0.01$. 


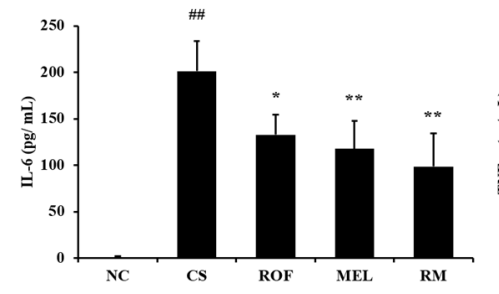

(a)

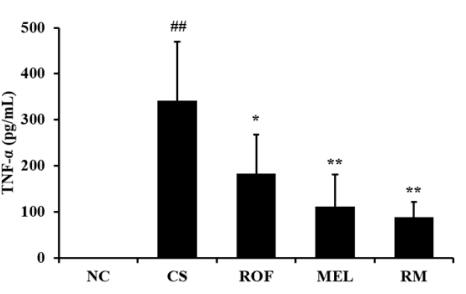

(b)

Figure 3. Melatonin decreased inflammatory cytokines in BALF of CS-exposed mice. The level of inflammatory cytokines was determined by ELISA assay. (a) Level of IL-6 and (b) level of TNF$\alpha$. NC: non-treated and non-exposure group; CS: PBS-treated and CS+LPS exposure group; ROF: roflumilast-treated and CS+LPS exposure group, MEL: melatonin-treated and CS+LPS exposure group, RM: roflumilast and melatonin co-treated and CS+LPS exposure group. The data are expressed as mean $\pm \mathrm{SD}(\mathrm{n}=5)$. ${ }^{\# \#}$ Significantly different from the NC group, $p<0.01 ;{ }^{*},{ }^{* *}$ significantly different from the CS group, $p<0.05$ and $p<0.01$, respectively.

\subsection{Effects of Melatonin on MMP-9, PDE4, and cAMP Levels in Mice Exposed to CS}

Mice in the CS group showed marked elevation in MMP-9 expression in lung tissues compared to mice from the NC group (Figure 4a). In contrast, the ROF, MEL, and RM groups showed a significant decrease in MMP-9 expression, with the RM group showing the most noticeable reduction. Further, the MMP-9 level was markedly increased in BALF in the CS group compared to the NC group (Figure $4 \mathrm{~b}$ ). However, ROF and MEL groups showed significantly lower MMP-9 level than the CS group. This reduction was more remarkable in the RM group. Consistently, PDE4B expression was markedly increased in the CS group compared to the NC group; however, the ROF and MEL groups showed significantly decreased PDE4B expression, compared to the CS group (Figure 4c). PDE4B expression was reduced to a much greater extent in the RM group than in the ROF or MEL groups. The cAMP level in BALF was markedly decreased in the CS group, compared to the NC group; however, it was significantly increased in ROF, MEL, and RM groups compared to the CS group (Figure 4d), with the RM group showing the greatest degree of elevation.
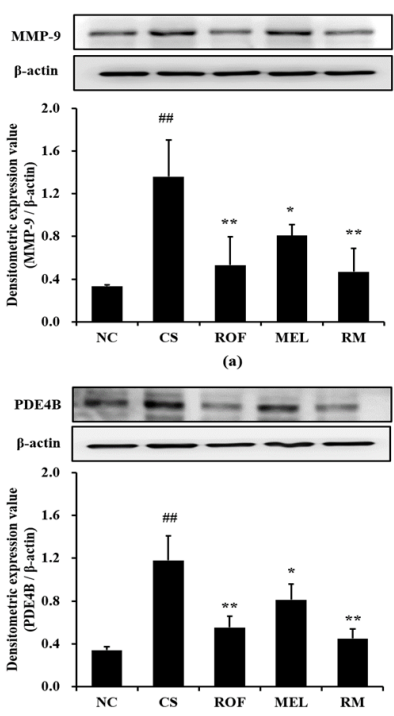

(c)
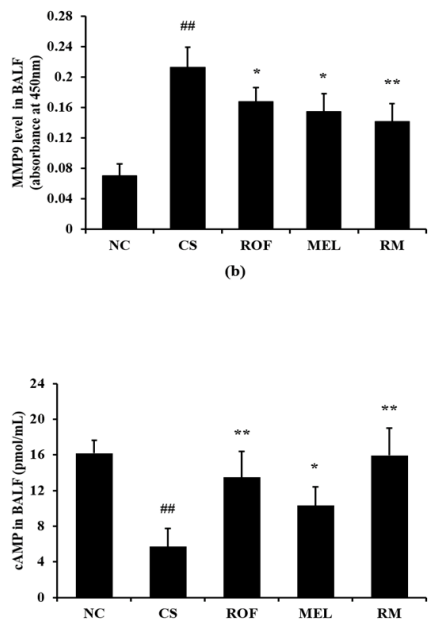

(d)

Figure 4. Melatonin reduced the elevation of MMP-9, PDE4B and CAMP in CS-exposed mice. (a) MMP-9 expression, (b) MMP-9 level in BALF, (c) PDE4B expression, (d) cAMP level in BALF. NC: non-treated and non-exposure group; CS: PBS-treated and CS+LPS exposure group; ROF: roflumilasttreated and CS+LPS exposure group, MEL: melatonin-treated and CS+LPS exposure group, RM: roflumilast and melatonin co-treated and CS+LPS exposure group. The data are expressed as mean $\pm \mathrm{SD}(\mathrm{n}=5)$. \#\# Significantly different from the NC group, $p<0.01{ }^{*},{ }^{* *}$ significantly different from the CS group, $p<0.05$ and $<0.01$, respectively. 


\subsection{Effects of Melatonin on Lung Function in Mice Exposed to CS}

The CS group showed markedly increased lung compliance than the NC group (Figure 5a). However, compared to the CS group, the ROF and MEL groups exhibited significant reduction in lung compliance. In particular, lung compliance was reduced to a greater extent in the RM group than in the ROF or MEL group. The CS group showed a significant reduction in elastance compared to the NC group (Figure 5b). In contrast, the ROF, MEL, and RM groups exhibited a marked elevation of elastance compared to the CS group, with the RM group showing the greatest degree of elevation.

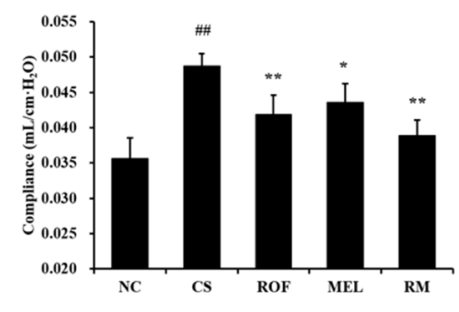

(a)

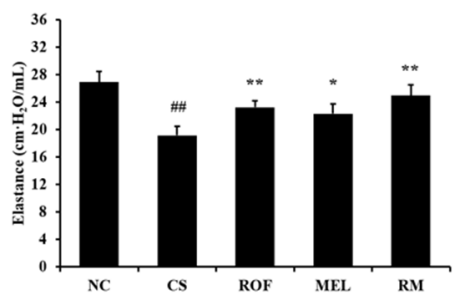

(b)

Figure 5. Melatonin restored worsened lung function in CS-exposed mice. (a) Compliance, (b) elastance. NC: non-treated and non-exposure group; CS: PBS-treated and CS+LPS exposure group; ROF: roflumilast-treated and CS+LPS exposure group, MEL: melatonin-treated and CS+LPS exposure group, RM: roflumilast and melatonin co-treated and CS+LPS exposure group. The data are expressed as mean $\pm \mathrm{SD}(\mathrm{n}=5)$. \#\# Significantly different from the NC group, $p<0.01$; $*, * *$ significantly different from the CS group, $p<0.05$ and $<0.01$, respectively.

\subsection{Effect of Melatonin on Inflammatory Mediators in CSC Stimulated NCI-H292 Cells}

Expression levels of IL-6 and TNF- $\alpha$ were significantly increased in CSC-stimulated cells compared to non-treated cells (Figure 6a,b, respectively). However, melatonin treatment significantly decreased the IL- 6 and TNF- $\alpha$ expression levels in CSC-stimulated cells. Roflumilast treatment also yielded similar results. Furthermore, roflumilast and melatonin cotreatment decreased the IL- 6 and TNF- $\alpha$ expression levels to a greater extent than roflumilast or melatonin treatment alone. Consistently, MMP-9 expression was higher in CSC-stimulated cells than in the non-treated cells (Figure 6c). By contrast, melatonin treatment significantly reduced MMP-9 expression, compared with CSC-stimulated cells. Similar results were obtained with roflumilast treatment. Moreover, this reduction was more enhanced in roflumilast and melatonin cotreated cells.

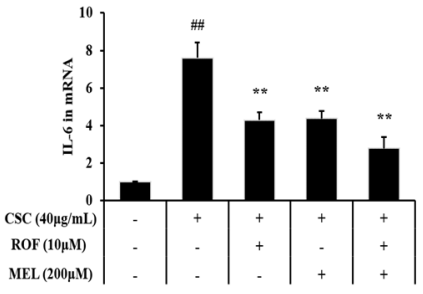

(a)

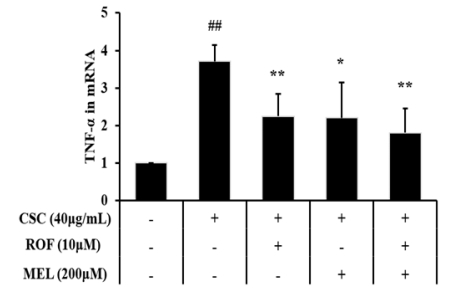

(b)

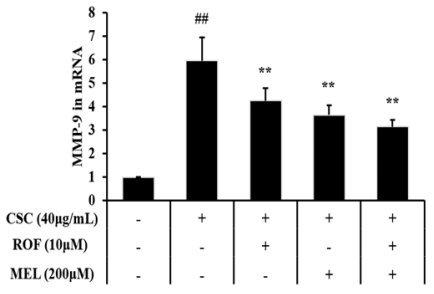

(c)

Figure 6. Melatonin reduced mRNA expression of inflammatory cytokines and MMP-9 in CSCstimulated cells. The level of mRNA expression of inflammatory cytokines and MMP-9 was determined by real-time PCR. (a) $I L-6$, (b) TNF- $\alpha$, (c) MMP-9. This experiment was repeatedly performed three times. ${ }^{\#}$ Significantly different from the non-treated cells, $p<0.01 ;{ }^{*},{ }^{* *}$ significantly different from the CSC-stimulated cells, $p<0.05$ and $<0.01$, respectively.

\subsection{Effect of Melatonin on PDE4B Expression and cAMP Activity in CSC-Stimulated NCI- H292 Cells}

Compared to non-treated cells, CSC-stimulated cells exhibited a significant increase in the PDE4B protein and mRNA expression levels (Figure 7a,b, respectively). However, melatonin treatment significantly suppressed the elevation of the PDE4B protein and mRNA 
expression in CSC-stimulated cells. Furthermore, roflumilast and melatonin cotreatment decreases the PDE4B protein and mRNA expression levels to a greater extent than roflumilast or melatonin treatment alone. Compared to the non-treated cells, the cAMP level was markedly reduced in CSC-stimulated cells (Figure 7c). However, melatonin treatment resulted in a significant increase in cAMP level, compared to CSC-stimulated cells. Similar results were obtained with roflumilast treatment. In addition, roflumilast and melatonin cotreatment resulted in the increase in cAMP levels; this increase was more than that obtained with roflumilast or melatonin treatment alone.

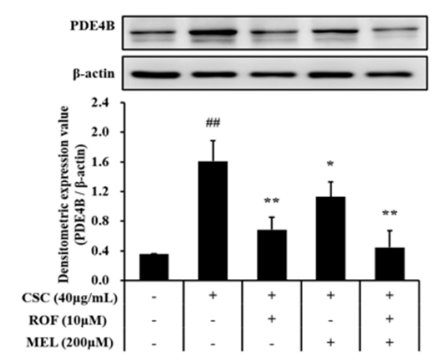

(a)

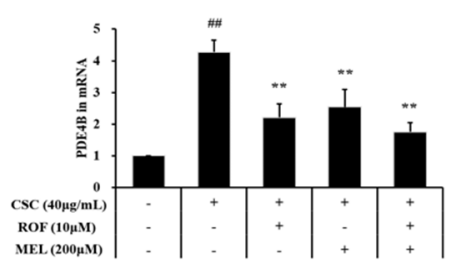

(b)

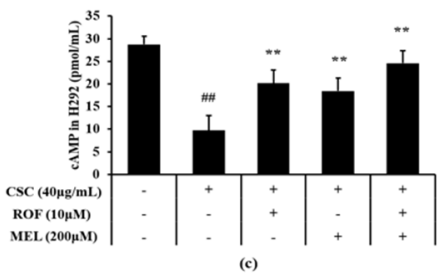

(c)

Figure 7. Melatonin decreased PDE4B and cAMP in CSC-stimulated cells. The protein and mRNA expression of PDE4B was determined in by immunoblotting and real-time PCR, respectively. cAMP level was determined in supernatant by ELISA. (a) PDE4B expression, (b) mRNA expression of $P D E 4 B,(\mathbf{c})$ level of cAMP. This experiment was repeatedly performed three times. \#\# Significantly different from the non-treated cells, $p<0.01 ;{ }^{*},{ }^{* *}$ significantly different from the CSC-stimulated cells, $p<0.05$ and $p<0.01$.

\section{Discussion}

Melatonin is used for the treatment of various diseases due to its pharmacological properties $[17,18]$. Although our previous study has shown that melatonin is effective in treating COPD, the exact mechanism of action of melatonin in the treatment of COPD is still very poorly understood [18]. In this study, we investigated the potential of melatonin to serve as a PDE4 inhibitor in the treatment of COPD using mice exposed to CS and CSC-stimulated cells. Melatonin effectively suppressed inflammatory cell infiltration, production of proinflammatory cytokines and expression of MMP-9 in mice exposed to CS and CSC-stimulated cells, which was accompanied with a reduction in PDE4 expression and elevation of cAMP levels. Due to these responses, melatonin ameliorated the CSexposure-induced worsening of lung function. In addition, these responses were further enhanced upon cotreatment with melatonin and roflumilast.

CS is considered a crucial contributor in the development of COPD [23-26]. Exposure to CS stimulated the recruitment of many inflammatory cells such as neutrophils and macrophages into lung tissues; these cells produce various inflammatory mediators including cytokines, reactive oxygen species, chemokines, and MMPs [25-28]. Because of these responses, the normal structure of the lung tissue is destroyed, resulting in declined lung function [26-28]. In our study, melatonin treatment inhibited the elevation of inflammatory cell count, cytokine levels, and MMP-9 expression in mice exposed to CS, with a reduction in the levels of inflammatory mediators in CSC-stimulated cells. Consistently, administration of melatonin effectively suppressed the recruitment of inflammatory cells into the lung tissues in mice exposed to CS. In particular, these responses were found to be pronounced upon cotreatment with melatonin and roflumilast. These results indicate that melatonin can effectively inhibit the development of COPD, and that cotreatment with roflumilast has a better therapeutic effect than the administration of either agent alone.

PDE4 inhibitors are clinically recommended drugs for the treatment of COPD [29]. PDE4 inhibitors inhibit the conversion of cAMP to AMP, thereby reducing inflammatory responses in the lung tissue and normalizing lung function [30,31]. During the development of COPD, exposure to CS increases the expression of PDE4 in the lung tissue, thereby reducing the levels of cAMP. This eventually worsens inflammatory responses in the 
lung tissue and causes a decrease in lung function [32,33]. Thus, PDE4 inhibitors are recognized as very important drugs in the treatment of COPD, and many researchers are currently focused on discovering COPD drugs, with an emphasis on PDE4 inhibition of candidate drugs [34-37]. In previous studies, PDE4 inhibitors reduced lung inflammation and restored lung function by increasing cAMP through inhibition of the PDE4 expression in CS-induced COPD animal models. In this study, administration of melatonin effectively reduced the expression of PDE4 caused upon CS exposure, increased the levels of cAMP, and led to the recovery of lung function following CS-exposure-induced decline in lung function. These responses were more noticeably detected upon cotreatment with melatonin and roflumilast. In addition, the same was observed in CSC-stimulated cells. Treatment with melatonin suppressed PDE4 expression in CSC-stimulated cells, with an elevation in the cAMP levels. This result indicated that the therapeutic effect of melatonin in COPD is associated with the suppression of PDE4, and when combined with the PDE4 inhibitor, its therapeutic effect in COPD is greatly elevated.

The synergistic effects of melatonin and roflumilast against COPD is considered to be related with its PDE4B inhibitory effect and other pharmacological properties, such as anti-inflammatory and antioxidative effects. There have been various experiments related to the anti-COPD effect of melatonin [18-22,38,39]. In previous studies, the anti-COPD effect of melatonin was associated with the anti-inflammatory and antioxidant effects of melatonin in in vivo and in vitro experiments using CS or CSC [18-21,38,39]. In this study, it is suggested that the anti-COPD effect of melatonin may be related not only to its anti-inflammatory and antioxidant properties, but also to the ability of inhibiting PDE4B expression. Therefore, it is considered to exhibit a synergistic effect when administered in combination with roflumilast due to the inhibitory effect of PDE4B expression and other pharmacological properties of melatonin.

In summary, treatment of melatonin attenuates inflammatory responses in CS-exposed mice and CSC-stimulated cells, which is correlated with elevation of cAMP via inhibition of PDE4 expression. Our results suggest that melatonin has potential as a PDE4 inhibitor in the treatment of COPD.

\section{Materials and Methods}

\subsection{Cell Culture and Viability Assay}

The NCI-H292 cell line was obtained from the ATCC (Manassas, VA, USA). Cells were cultured in RPMI 1640 (WELGENE, Gyeongsangbuk-do, Republic of Korea) supplemented with $10 \%$ heat-inactivated fetal bovine serum and antibiotics (WELGENE). The viability assay was performed using 3-(4,5-dimethylthiazol-2-yl)-2,5-diphenyltetrazolium bromide (MTT, Sigma-Aldrich, Saint Louis, MO, USA). Cells were maintained in 96-well plates at a density of $3 \times 10^{4}$ cells/well. Roflumilast (ROF, Sigma-Aldrich) and melatonin (MEL, Sigma-Aldrich) were added to each well as follows: ROF: 2.5, 5, 10, and $20 \mu \mathrm{M}$; MEL: 100, 200, 400, and $800 \mu \mathrm{M}$. This was followed by incubation for $24 \mathrm{~h}$. MTT solution $(10 \mu \mathrm{L})$ was added to each well, and the cells were incubated for $4 \mathrm{~h}$ at $37^{\circ} \mathrm{C}$; then, $100 \mu \mathrm{L}$ of dimethyl sulfoxide (DMSO, Sigma-Aldrich) was added to each well to solubilize the formazan produced. The optical density of the culture was measured at $570 \mathrm{~nm}$.

\subsection{RNA Isolation and Real-Time PCR}

NCI-H292 cells were seeded on $60 \mathrm{~mm}$ dishes at a density of $1 \times 10^{6}$ cells $/$ well and were treated with ROF $(10 \mu \mathrm{M})$ and MEL $(200 \mu \mathrm{M})$, for 1 hour. After incubation, cells were stimulated with CSC. CSC was prepared as previously described [18]. Total RNA was isolated using RNA isolation kit (Invitrogen, Carlsbad, CA, USA). cDNA was synthesized using Oligo DTs and the cDNA synthesis kit (Qiagen, Hilden, Germany). Polymerase chain reactions were performed using specific forward and reverse primers (TNF- $\alpha$, forward, 5'-CAAAGTAGACCTGCCCAGAC-3' , reverse, 5' - GACCTCTCTCTAATCAGCCC-3 ${ }^{\prime}$; IL-6, forward, 5' -ATGCAATAACCACCCCTGAC-3' and reverse, $5^{\prime}$ - ATCTGAGGTGCCCATG CTAC-3'; MMP-9, forward, 5' - AAGGGCGTCGTGGTTCCAACTC- $3^{\prime}$ and reverse, 5' - AGCA 
TTGCCGTCCTGGGTGTAG-3'; PDE4B, forward, 5' -ATTGTAGCAATGGACAGAC-3' and reverse, $5^{\prime}$-GTATCGAGATCCTGAGCATC-3' ; GAPDH, forward, $5^{\prime}$ - CAAAAG GGTCATCT CTG-3', reverse, $5^{\prime}$ - CCTGCTTCACCACCTTCTTG-3'). The mRNA expression levels of the target genes were normalized to that of housekeeping gene GAPDH.

\subsection{CS Induced Airway Inflammation}

Male C57BL/ 6 mice (6-8 weeks old, 20-25g) were purchased from Semtaco Co (Osan, Korea). Mice were housed in standard conditions. All procedures were approved by the Institutional Animal Care and Use Committee of the Chonnam National University (CNU IACUC-YB-R-2016-18). CS-induced airway inflammation model was established as explained in a previous study [40]. Briefly, mice were exposed to 3R4F research cigarettes (Kentucky reference cigarette, University of Kentucky, USA) for 14 days using a CS generator (Daehan Biolink, Inchun, Korea) and LPS ( $5 \mu \mathrm{g} /$ mouse, Sigma-Aldrich) was administered intranasally on day 5 . ROF $(10 \mathrm{mg} / \mathrm{kg})$ and MEL $(30 \mathrm{mg} / \mathrm{kg})$ were intraperitoneally administered 1 hour before exposure to CS for 14 days. Mice were randomly divided into five groups as follows $(n=5)$ : NC (normal control; no treatment + fresh air and PBS exposure), CS (PBS treatment + CS and LPS exposure), ROF (roflumilast treatment + CS and LPS exposure), MEL (melatonin treatment + CS and LPS exposure), and RM (roflumilast and melatonin treatment + CS and LPS exposure). Lung function was evaluated using Flexivent (SCIREQ Inc., Montreal, QC, Canada) $24 \mathrm{~h}$ after the last CS exposure. The animals were subjected to tracheostomy, and detection probe was inserted into trachea. The compliance and elastance of each animal were evaluated according to the programmed protocols of the instrument.

\subsection{Bronchoalveolar Lavage Fluid (BALF) Collection and Analysis}

BALF collection was performed as described previously [12]. Animals were subjected to tracheostomy, and cold PBS $(0.7 \mathrm{~mL})$ was infused into the lung tissue and withdrawn via tracheal cannulation twice (total, $1.4 \mathrm{~mL}$ ). The BALF thus obtained was centrifuged at a speed of $1500 \mathrm{rpm}$ for $5 \mathrm{~min}$, and then the supernatant was used to evaluate cytokine and cAMP activity. Cell pellets were used to determine inflammatory cell counts in the BALF. To evaluate differential cell count, cells were attached on slides and stained with Diff-Quik reagent (IMEB, Deerfield, IL, USA). Total cell count was determined using an automatic cell count analyzer (Thermo Fisher Scientific, San Diego, CA, USA). Levels of cytokines (R\&D System, Minneapolis, MN, USA) and activity of cAMP (Abcam, Cambridge, UK) were evaluated using an ELISA kit according to the manufacturer's instructions.

\subsection{Immunoblotting}

The lung tissue was homogenized $(1 / 10 w / v)$ using a homogenizer in tissue lysis/extraction reagent (Sigma-Aldrich) supplemented with protease inhibitors (SigmaAldrich). Immunoblotting was performed as described previously [12]. The following primary antibodies were used: anti-PDE4B (1:1000 dilution; Abcam), anti-MMP-9 (1:1000 dilution; Abcam), and anti- $\beta$-actin (1:1000 dilution; Cell signaling, Denver, MA, USA). Expression of PDE4B, MMP-9, and $\beta$-actin were evaluated using ChemiDoc (Bio-Rad, Hercules, CA, USA).

\subsection{Histological Analysis}

The right lungs of mice from each group were fixed in $10 \%$ buffered formalin (SigmaAldrich) for 3 days at room temperature. Fixed lung tissue was embedded in paraffin blocks, cut into $4 \mu \mathrm{m}$ thick sections, deparaffinized using xylene, and dehydrated using ethanol. Following a $5 \mathrm{~min}$ wash with distilled water, the tissue sections were stained using hematoxylin and eosin stain to evaluate inflammatory cell infiltration. Quantification of inflammatory responses was conducted using an image analyzer (IMT i-Solution Ins., Vancouver, BC, Canada). 


\subsection{Statistical Analysis}

Data are presented as the means \pm standard deviation (SD). Statistical significance of results was determined using ANOVA followed by the multiple comparison test with Dunnet's adjustment and were considered significant at $p<0.05$.

Author Contributions: Conceptualization, I.-S.S.; methodology, J.-O.L. and S.-J.L.; formal analysis, J.-O.L., S.-J.L., S.-W.P., Y.-K.C. and W.-I.K.; investigation, J.-O.L. and S.-W.P.; resources, S.-J.L. and W.-I.K.; data curation, J.-S.K. and I.-S.S.; writing-original draft preparation, J.-O.L. and W.-I.K.; writing-review and editing, J.-C.K. and I.-S.S.; supervision, I.-S.S.; project administration, W.-I.K. and J.-O.L.; funding acquisition, I.-S.S. All authors have read and agreed to the published version of the manuscript.

Funding: This research was supported by a National Research Foundation of Korea (NRF) grant funded by the Korea Government (MSIT) (grant number: NRF-2019R1A2C4070145).

Institutional Review Board Statement: All experiments were conducted according to a protocol approved by the Chonnam National University (CNU) Institutional Animal Care and Use Committee. (CNU IACUC-YBR-2016-18, Gwangju, South Korea).

Informed Consent Statement: Not applicable.

Data Availability Statement: Data is contained within the article.

Conflicts of Interest: The authors declare no conflict of interest.

\section{References}

1. Quaderi, S.A.; Hurst, J.R. The unmet global burden of COPD. Glob. Health Epidemiol. Genom. 2018, 3, e4. [CrossRef] [PubMed]

2. Groneberq, D.A.; Chung, K.F. Models of chronic obstructive pulmonary disease. Respir. Res. 2004, 5, 18. [CrossRef] [PubMed]

3. Strzelak, A.; Ratajczak, A.; Adamiec, A.; Feleszko, W. Tobacco smoke induces and alters immune responses in the lung triggering inflammation, allergy, asthma and other lung diseases: A mechanistic review. Int. J. Environ. Res. Public Health 2018, 15, 1033. [CrossRef] [PubMed]

4. Pang, M.; Yuan, Y.; Wang, D.; Li, T.; Wang, D.; Shi, X.; Guo, M.; Wang, C.; Zhang, X.; Zheng, G.; et al. Recombinant CC16 protein inhibits the production of pro-inflammatory cytokines via NF- $\mathrm{BB}$ and p38 MAPK pathways in LPS-activated RAW264.7 macrophages. Acta Biochim. Biophys. Sin. 2017, 49, 435-443. [CrossRef]

5. Yan, K.; Gao, L.N.; Cui, Y.L.; Zhang, Y.; Zhou, X. The cyclic AMP signaling pathway: Exploring targets for successful drug discovery (Review). Mol. Med. Rep. 2016, 13, 3715-3723. [CrossRef]

6. Omori, K.; Kotera, J. Overview of PDEs and their regulation. Circ. Res. 2007, 100, 309-327. [CrossRef]

7. Jacob, C.; Szilagyi, C.; Allen, J.M.; Bertrand, C.; Lagente, V. Role of PDE4 in superoxide anion generation through p44/42MAPK regulation: A cAMP and a PKA-independent mechanism. Br. J. Pharmacol. 2004, 143, 257-268. [CrossRef]

8. Zuo, H.; Han, B.; Poppinga, W.J.; Ringnalda, L.; Kistemaker, L.E.M.; Halayko, A.J.; Gosens, R.; Nikolaev, V.O.; Schmidt, M. Cigarette smoke up-regulates PDE3 and PDE4 to decrease cAMP in airway cells. Br. J. Pharmacol. 2018, 175, 2988-3006. [CrossRef]

9. De Visser, Y.P.; Walther, F.J.; Laghmani, E.H.; van Wijngaarden, S.; Nieuwland, K.; Wagenaar, G.T. Phosphodiesterase-4 inhibition attenuates pulmonary inflammation in neonatal lung injury. Eur. Respir. J. 2008, 31, 633-644. [CrossRef]

10. Tashkin, D.P. Roflumilast: The new orally active, selective phophodiesterase-4 inhibitor, for the treatment of COPD. Expert Opin. Pharmacother. 2014, 15, 85-96. [CrossRef]

11. Park, E.; Jeon, W.Y.; Seo, C.S.; Ha, H.; Jin, S.E.; Kim, J.; Lee, M.Y. Yukgunja-tang, a traditional herbal formula, attenuates cigarette smoke-induced lung inflammation in a mouse model. Pharmacogn. Mag. 2018, 14, 275-282.

12. Shin, N.R.; Park, J.W.; Lee, I.C.; Ko, J.W.; Park, S.H.; Kim, J.S.; Kim, J.C.; Ahn, K.S.; Shin, I.S. Melatonin suppresses fibrotic responses induced by cigarette smoke via downregulation of TGF- $\beta 1$. Oncotarget 2017, 8, 95692-95703. [CrossRef]

13. Li, Y.; Li, S.; Zhou, Y.; Meng, X.; Zhang, J.J.; Xu, D.P.; Li, H.B. Melatonin for the prevention and treatment of cancer. Oncotarget 2017, 8, 39896-39921. [CrossRef]

14. Slominski, A.T.; Zmijewski, M.A.; Semak, I.; Kim, T.K.; Janjetovic, Z.; Slominski, R.M.; Zmijewski, W. Melatonin, mitochondria, and the skin. Cell. Mol. Life Sci. 2017, 74, 3913-3925. [CrossRef]

15. Kim, T.K.; Kleszczynski, K.; Janjetovic, Z.; Sweatman, T.; Lin, Z.; Li, W.; Reiter, R.J.; Fischer, T.W.; Slominski, A.T. Metabolism of melatonin and biological activity of intermediates of melatoninergic pathway in human skin cells. FASEB J. 2013, 27, $2742-2755$. [CrossRef]

16. Slominski, A.T.; Semak, I.; Fischer, T.M.; Kim, T.K.; Kleszczynski, K.; Hardeland, R.; Reiter, R.J. Metabolism of melatonin in the skin: Why is it important? Exp. Dermatol. 2017, 26, 563-568. [CrossRef]

17. Shin, I.S.; Park, J.W.; Shin, N.R.; Jeon, C.M.; Kwon, O.K.; Lee, M.Y.; Kim, H.S.; Kim, J.C.; Oh, S.R.; Ahn, K.S. Melatonin inhibits MUC5AC production via suppression of MAPK signaling in human airway epithelial cells. J. Pineal Res. 2014, 56, 398-407. [CrossRef] 
18. Shin, I.S.; Shin, N.R.; Park, J.W.; Jeon, C.M.; Hong, J.M.; Kwon, O.K.; Kim, J.S.; Lee, I.C.; Kim, J.C.; Oh, S.R.; et al. Melatonin attenuates neutrophil inflammation and mucus secretion in cigarette smoke-induced chronic obstructive pulmonary diseases via the suppression of Erk-Sp1 signaling. J. Pineal Res. 2015, 58, 50-60. [CrossRef]

19. He, B.; Zhang, W.; Qiao, J.; Peng, Z.; Chai, X. Melatonin protects against COPD by attenuating apoptosis and endoplasmic reticulum stress via upregulating SIRT1 expression in rats. Can. J. Physiol. Pharmacol. 2019, 97, 386-391. [CrossRef]

20. Shin, N.R.; Ko, J.W.; Kim, J.C.; Park, G.; Kim, S.H.; Kim, M.S.; Kim, J.S.; Shin, I.S. Role of melatonin as an SIRT1 enhancer in chronic obstructive pulmonary disease induced by cigarette smoke. J. Cell. Mol. Med. 2020, 24, 1151-1156. [CrossRef]

21. Kim, G.D.; Lee, S.E.; Kim, T.H.; Jin, Y.H.; Park, Y.S.; Park, C.S. Melatonin suppresses acrolein-induced IL-8 production in human pulmonary fibroblast. J. Pineal Res. 2021, 52, 356-364. [CrossRef]

22. De Matos Cavalcnte, A.G.; de Bruin, P.F.; de Bruin, V.M.; Nunes, D.N.; Pereira, E.D.; Cavalcante, M.M.; Andrade, G.M. Melatonin reduced lung oxidative stress in patients with chronic obstructive pulmonary disease: A randomized, double-blind, placebocontrolled study. J. Pineal Res. 2012, 53, 238-244. [CrossRef]

23. Devine, J.F. Chronic obstructive pulmonary disease: An overview. Am. Health Drug Benefits 2008, 1, 34-42.

24. Nam, H.S.; Izumchenko, E.; Dasgupta, S.; Hoque, M.O. Mitochondria in chronic obstructive pulmonary disease and lung cancer: Where are we now? Biomark. Med. 2017, 11, 475-789. [CrossRef]

25. Cornwell, W.D.; Kim, V.; Song, C.; Rogers, T.J. Pathogenesis of inflammation and repair in advanced COPD. Semin. Respir. Crit. Care Med. 2010, 31, 257-266. [CrossRef]

26. Pan, Z.; Yu, H.; Liao, J.L. Probing cellular and molecular mechanisms of cigarette smoke-induced immune response in the progression of chronic obstructive pulmonary disease using multiscale network modeling. PLoS ONE 2016, 11, e0163192. [CrossRef]

27. O'Donnell, R.; Breen, D.; Wilson, S.; Djukanovic, R. Inflammatory cells in the airways in COPD. Thorax 2006, 61, 448-454. [CrossRef]

28. Wang, Y.; Xu, J.; Meng, Y.; Adcock, I.M.; Yao, X. Role of inflammatory cells in airway remodeling in COPD. Int. J. Chron. Obstruct. Pulmon. Dis. 2018, 13, 3341-3348. [CrossRef]

29. Li, H.; Zuo, J.; Tang, W. Phosphodiesterase-4 inhibitors for the treatment of inflammatory diseases. Front. Pharmacol. 2018, 9 , 1048. [CrossRef]

30. Perez-Aso, M.; Montesinos, M.C.; Mediero, A.; Wilder, T.; Schafer, P.H.; Cronstein, B. Apremilast, a novel phosphodiesterase 4 (PDE4) inhibitor, regulates inflammation through multiple cAMP downstream effectors. Arthritis Res. Ther. 2015, 17, 249. [CrossRef]

31. Brown, W.M. Treating COPD with PDE 4 inhibitors. Int. J. Chron. Obstruct. Pulmon. Dis. 2007, 2, 517-533. [PubMed]

32. Mclvor, R.A. Future options for disease intervention: Important advances in phosphodiesterase 4 inhibitors. Eur. Respir. Rev. 2007, 16, 105-112. [CrossRef]

33. Demizu, S.; Asaka, N.; Kawahara, H.; Sasaki, E. TAS-203, an oral phosphodiesterase 4 inhibitor, exerts anti-inflammatory activities in a rat airway inflammation model. Eur. J. Pharmacol. 2019, 849, 22-29. [CrossRef] [PubMed]

34. Seimetz, M.; Parajuli, N.; Pichl, A.; Bednorz, M.; Ghofrani, H.A.; Schermuly, R.T.; Seeger, W.; Grimminger, F.; Weissmann, N. Cigarette smoke-induced emphysema and pulmonary hypertension can be prevented by phosphodiesterase 4 and 5 inhibition in mice. PLoS ONE 2015, 10, e0129327. [CrossRef]

35. Kubo, S.; Kobayashi, M.; Iwata, M.; Takahashi, K.; Miyata, K.; Shimizu, Y. Disease-modifying effect of ASP3258, a novel phosphodiesterase type 4 inhibitor, on subchronic cigarette smoke exposure-induced lung injury in guinea pigs. Eur. J. Pharmacol. 2011, 659, 79-84. [CrossRef]

36. Martorana, P.A.; Beume, R.; Lucattelli, M.; Wollin, L.; Lungarella, G. Roflumilast fully prevents emphysema in mice chronically exposed to cigarette smoke. Am. J. Respir. Crit. Care Med. 2005, 172, 848-853. [CrossRef]

37. Wan, W.Y.; Morris, A.; Kinnear, G.; Pearce, W.; Mok, J.; Wyss, D.; Stevenson, C.S. Pharmacological characterisation of antiinflammatory compounds in acute and chronic mouse models of cigarette smoke-induced inflammation. Respir. Res. 2010, 11, 126. [CrossRef]

38. Mahalanobish, S.; Dutta, S.; Saha, S.; Sil, P.C. Melatonin induced suppression of ER stress and mitochondrial dysfunction inhibited NLRP3 inflammasome activation in COPD mice. Food Chem. Toxicol. 2020, 144, 111588. [CrossRef]

39. Peng, Z.; Zhang, W.; Qiao, J.; He, B. Melatonin attenuates airway inflammation via SIRT1 dependent inhibition of NLRP3 inflammasome and IL-1beta in rats with COPD. Int. Immunopharmacol. 2018, 62, 23-28. [CrossRef]

40. Ko, J.W.; Seo, C.S.; Shin, N.R.; Kim, J.S.; Lee, S.I.; Kim, J.C.; Kim, S.H.; Shin, I.S. Modificated Mahuang-Tang, a traditional herbal medicine suppresses inflammatory responses induced by cigarette smoke in human airway epithelial cell and mice. Phytomedicine 2019, 59, 152777. [CrossRef] 